> Le diabète de type 1 est une maladie autoimmune résultant de la destruction des cellules $\beta$ pancréatiques par le système immunitaire. Les lymphocytes NKT (natural killer $T$ cell) sont des cellules régulatrices qui inhibent le développement du diabète de type 1 dans les modèles murins, tout en favorisant les réponses contre les agents infectieux. Les mécanismes régulateurs impliquent la production de cytokines et des modifications fonctionnelles des cellules dendritiques. La sélection de molécules ciblant spécifiquement les lymphocytes NKT représente une étape importante pour l'utilisation thérapeutique de ces lymphocytes afin d'inhiber le développement du diabète de type 1 chez les patients. <

Le diabète de type 1 est une maladie auto-immune résultant de la destruction des cellules $\beta$ pancréatiques productrices d'insuline par les cellules du système immunitaire. Cette maladie est caractérisée par la présence d'auto-anticorps et de lymphocytes T autoréactifs dirigés contre des antigènes produits par les cellules $\beta$ pancréatiques. Bien que de nombreuses études soient consacrées à la caractérisation de ces réponses autoimmunes, il devient de plus en plus évident que d'autres cellules du système immunitaire jouent un rôle déterminant dans le développement du diabète de type 1 [ 1 , 2]. L'étude des mécanismes conduisant à l'apparition de cette pathologie a révélé le rôle crucial des mécanismes de régulation immunitaire. Parmi les cellules régulatrices, une population particulière, les lymphocytes NKT (natural killer $T$ cell), pourrait devenir une cible thérapeutique de choix dans la prévention du diabète de type 1 .

\section{Les cellules NKT}

Les cellules NKT sont des lymphocytes T non conventionnels, car ils ne reconnaissent pas les molécules du CMH (complexe majeur d'histocompatibilité), mais la molé-

Cet article fait partie du numéro thématique « Diabètes : approches thérapeutiques émergentes ».

\title{
Diabète : \\ approches thérapeutiques émergentes \\ Rôle régulateur \\ des lymphocytes \\ NKT dans la \\ prévention du \\ diabète de type 1
}

Liana Ghazarian ${ }^{1,2,3 *}$, Yannick Simoni ${ }^{1,2,3 *}$, Karine

Pingris $^{1,3}$, Lucie Beaudoin ${ }^{1,2,3}$, Agnès Lehuen ${ }^{1,2,3,4}$

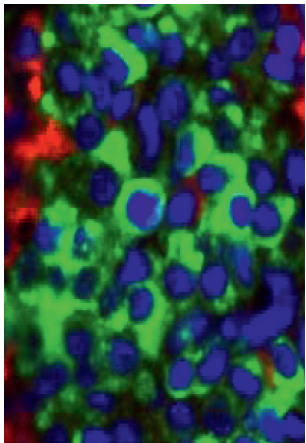

cule non polymorphe CDld. Le terme NKT reflète leurs caractéristiques phénotypiques et fonctionnelles partagées avec des cellules natural killer (NK), comme l'expression du marqueur NK1.1 ou CD16l chez l'homme. Les lymphocytes NKT sont considérés comme des lymphocytes T innés ayant un phénotype de cellules activées/effectrices, et ils sont très conservés chez la souris et chez l'homme [3]. Alors que les molécules classiques du CMH (comme les molécules HLA [human leukocyte antigen]-DR) présentent des antigènes peptidiques aux lymphocytes $T$, les molécules non classiques du CMH (comme le CDId) présentent des antigènes non peptidiques [3]. De nombreux travaux ont mis en évidence le rôle régulateur des lymphocytes NKT, et particulièrement leur capacité à inhiber le développement du diabète de type 1 dans les modèles murins [4]. Les cellules NKT modulent les réponses immunitaires en produisant rapidement de grandes quantités de cytokines et en induisant l'expression de différentes molécules de surface. Elles influencent le développement des réponses immunitaires innées et adaptatives. Classiquement, les lymphocytes NKT sont divisés en trois groupes: NKT invariants (iNKT), NKT de type II et NKT-like, selon l'expression de leur récepteur T (TCR) et leur spécificité antigénique (Figure 1) [4].

Les lymphocytes iNKT expriment un TCR $\alpha$ invariant, V $\alpha 14-J \alpha 18$ chez la souris et V $\alpha 24-J \alpha 18$ chez l'homme, et reconnaissent des glycolipides 


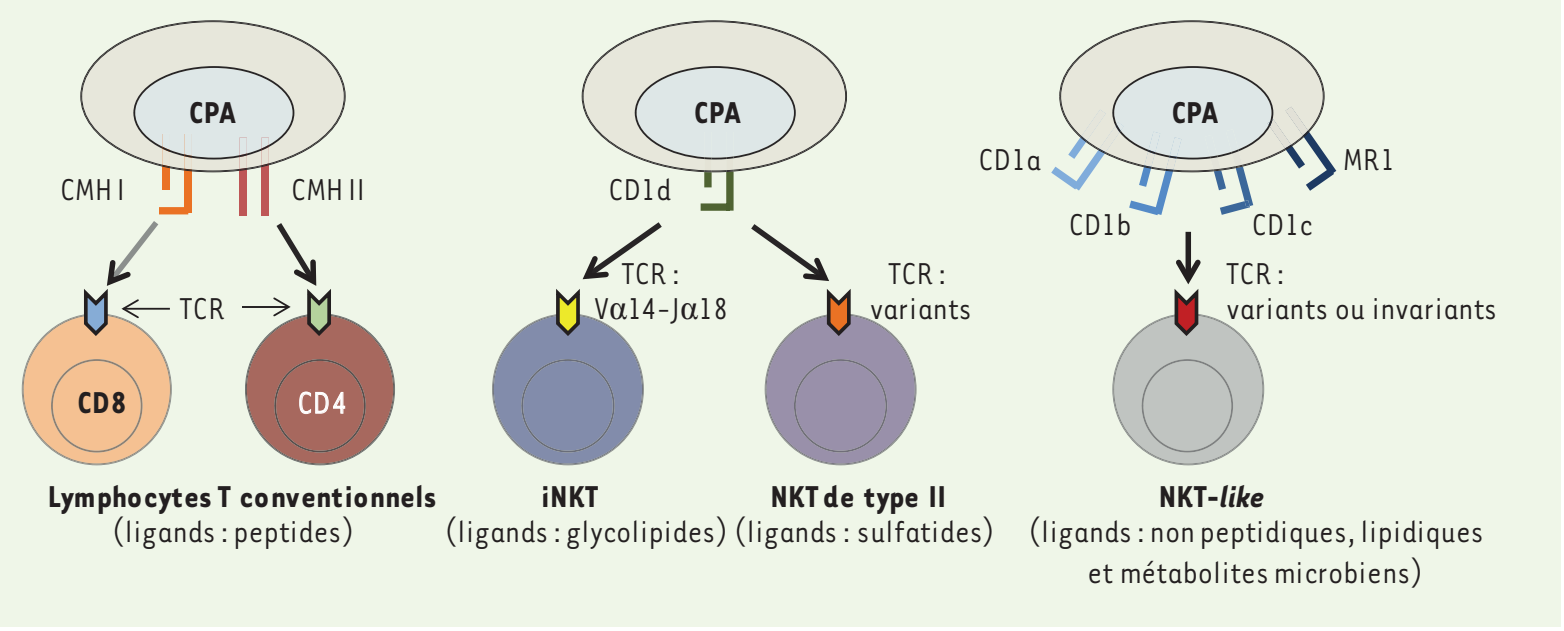

Figure 1. Lymphocytes T et lymphocytes NKT. Alors que lymphocytes T conventionnels reconnaissent des peptides présentés par le CMH I ou CMH II (gauche), les lymphocytes NKT expriment un TCR restreint par les molécules non classiques du CMH. Les lymphocytes iNKT et NKT de type II sont restreints par la molécule CDld qui présente des glycolipides ou des sulfatides (centre). Les lymphocytes NKT-like sont restreints par d'autres molécules non polymorphes comme CDla, b, c ou MRI (MHC related protein 1) (droite). CPA : cellules présentatrices d'antigène ; CMH : complexe majeur d'histocompatibilité ; TCR : $T$ cell receptor.

présentés par CDld, en particulier l' $\alpha$-galactosylcéramide ( $\alpha$ GalCer). Les lymphocytes NKT de type II sont également restreints par la molécule CDId, mais ils ne reconnaissent pas l' $\alpha$ GalCer et ils expriment un TCR $\alpha$ variable [3]. Parmi cette population, certains lymphocytes reconnaissent des sulfatides ou le lysophosphatidylcholine. Plus récemment, une autre population de lymphocytes $T \alpha \beta$ non conventionnels, reconnaissant la molécule non polymorphe MRI (MHC-related protein 1), a été caractérisée. Les souris transgéniques $V \alpha 14-J \alpha 18$ et les outils permettant la détection spécifique des lymphocytes iNKT (tétramères CDld- $\alpha$ GalCer et anticorps 6Bl1) ont permis une étude approfondie de ces cellules et, en particulier, de déterminer leur rôle régulateur dans le diabète de type 1.

\section{Les lymphocytes iNKT dans le diabète de type 1}

L'implication des lymphocytes iNKT dans la régulation de maladies auto-immunes a été proposée suite à l'observation d'anomalies de cette population cellulaire chez les patients affectés de cette pathologie, ainsi que chez les modèles animaux correspondants. Le rôle régulateur des cellules iNKT dans le diabète de type $l$ a été démontré puis analysé grâce aux souris génétiquement modifiées qui sont, soit dépourvues en lymphocytes NKT, soit au contraire expriment une fréquence accrue de cette population lymphocytaire.

\section{La fréquence des lymphocytes iNKT}

Le modèle animal le plus utilisé pour l'étude du diabète de type 1 est la souris NOD (non obese diabetic), car elle présente des signes cliniques similaires à ceux observés chez l'homme [1]. Les souris femelles NOD développent spontanément un diabète à partir de 12-15 semaines d'âge. La caractérisation des lymphocytes iNKT chez ces souris a montré une fréquence et un nombre plus faibles de cellules iNKT dans le thymus et la rate par rapport aux souches de souris contrôles (BALB/c, C57BL/6, $A K R$ et NOR) $[5,6]$. De plus, notre équipe a montré que l'augmentation du nombre des lymphocytes iNKT, provoquée par l'expression de la chaîne Val4-Jol8 par transgenèse, diminue l'incidence du diabète des souris NOD [7]. L'incidence du diabète diminue également quand les cellules iNKT s'accumulent dans les ganglions drainants le pancréas suite à la surexpression de la molécule CDld par les cellules $\beta$ pancréatiques [8]. Inversement, l'incidence du diabète est plus élevée et accélérée chez les souris déficientes en cellules NKT (souris NOD $\mathrm{CDld}^{-/-}$) comparées aux souris NOD sauvages $[9,10]$.

\section{Le rôle fonctionnel des cellules iNKT}

Le développement du diabète est associé à la présence de lymphocytes $T$ anti-cellules $\beta$ pancréatiques de type Thl, alors que la protection contre cette pathologie est fréquemment associée à des réponses de type Th2 [1]. Bien que les cellules iNKT puissent produire diverses cytokines dont I'IFN $\gamma$ (interféron $\gamma$ ) et I'IL-4 (interleukine-4), de nombreuses études ont montré que leur production d'IL-4 inhibe les réponses auto-immunes pathogènes de type Thl (Figure 2). Dans ce contexte, il est intéressant de noter que les cellules iNKT des souris NOD produisent moins d'IL-4 que celles de souris non diabétiques, comme les souris $B A L B / c$ [5]. En revanche, la correction d'un déficit en 


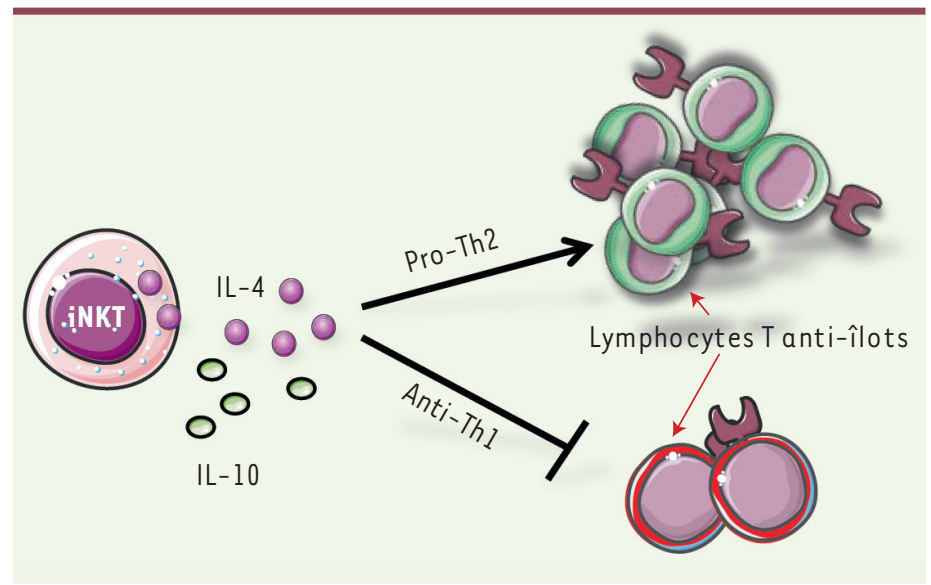

Figure 2. Rôle protecteur des cytokines produites par les lymphocytes iNKT. Les lymphocytes iNKT peuvent inhiber le développement du diabète de type 1 par la production de cytokines IL-4 et/ou IL-10. Ces deux cytokines inhibent les réponses Thl diabétogènes, et l'IL-4 favorise les réponses Th2, non pathogènes dans le diabète.

IL-4 par l'administration d'IL-4 exogène [11] ou l'expression d'IL-4 sous le contrôle du promoteur de l'insuline prévient l'apparition du diabète chez les souris NOD. De façon similaire, nous avons montré que la protection du diabète chez les souris V $\alpha 14-J \alpha 18$ transgéniques est associée à une augmentation de la production d'IL-4 et une diminution de celle d'IFN $\gamma$ localement dans le pancréas [12]. Une autre étude confirme le rôle des cytokines dans la protection du diabète par des thymocytes $\alpha / \beta^{-} \mathrm{TCR}^{+} \mathrm{CD} 4^{-} \mathrm{CD} 8^{-}$, fortement enrichis en cellules iNKT. Dans ce système, l'effet protecteur des cellules iNKT est dépendant de I'IL-4 et de I'IL-10, car l'administration d'anticorps neutralisant I'IL-4 et I'IL-10 abolit la protection contre le diabète [13].

Il est important de noter que les cellules iNKT peuvent également inhiber le développement du diabète par d'autres mécanismes d'immunorégulation indépendants de l'IL-4 et de l'IL-10. Des expériences de transfert de populations monoclonales de lymphocytes $T$ diabétogènes, soit $\mathrm{CD} 4^{+}$, soit $C D 8^{+}$, ont révélé la capacité des cellules iNKT à inhiber la différenciation de ces lymphocytes $T$ anti-cellules $\beta$ pancréatiques en cellules effectrices (Figure 3). Dans ces études, les lymphocytes T autoréactifs ne dévient pas vers une réponse Th2, mais ils deviennent anergiques ou non fonctionnels [14, 15]. L'activation abortive des lymphocytes T anticellules $\beta$ pancréatiques, en présence de lymphocytes iNKT, est associée à des anomalies des cellules dendritiques dans les ganglions pancréatiques $[14,16]$. Nous analysons actuellement les capacités régulatrices des lymphocytes T anti-cellules $\beta$ pancréatiques anergiques. L'inhibition de l'activation des lymphocytes CD4 autoréactifs nécessite un contact cellulaire entre ces lymphocytes, les cellules iNKT et les cellules présentatrices d'antigènes $[17,18]$. Bien que ces contacts cellulaires ne soient pas dépendants de la molécule $C D I d$, la surexpression par transgenèse de cette molécule à la surface des cellules $\beta$ pancréatiques induit l'accumulation dans les ganglions drainants des cellules iNKT, qui inhibent l'activation des lymphocytes T autoréactifs [8].
Malgré le rôle protecteur des lymphocytes iNKT dans leur globalité, une sous-population de cellules iNKT produisant de I'IL-17 joue un rôle délétère dans le diabète. En effet, une nouvelle sous-population de lymphocytes iNKT (CD4- NKI.1-) producteurs d'IL-17 a été récemment décrite [19]. Nous avons observé que les souris NOD possèdent un nombre plus élevé de ces cellules iNKT17 par rapport aux souches contrôles (C57BL/6 et $B A L B / c$ ) [20]. De plus, des expériences de transfert ont montré que ces cellules accentuent le développement du diabète induit par les lymphocytes T CD4 diabétogènes, alors que la population iNKT CD4 ${ }^{+} N K 1.1^{+}$, dépourvue de cellules NKT17, prévient très efficacement le diabète (Figure 4).

Les lymphocytes iNKT chez les patients diabétiques

Différentes études chez des patients présentant diverses pathologies auto-immunes ont montré le lien entre des anomalies des lymphocytes iNKT et une dérégulation du système immunitaire [4]. Une première étude, publiée dans Nature en 1998, a décrit des défauts numériques et fonctionnels des lymphocytes iNKT chez des patients diabétiques comparés à des sujets contrôles [21]. De plus, il a été observé un défaut de la sous-population $\mathrm{CD}^{+}$des lymphocytes iNKT dans le sang des patients diabétiques [22]. Contrairement à la souris, la présence des lymphocytes iNKT17 n'a pas encore été décrite parmi les lymphocytes circulants chez l'homme, bien que ceux-ci puissent être obtenus dans certaines conditions de culture in vitro. L'analyse des lymphocytes iNKT dans les ganglions pancréatiques a révélé un défaut de production d'IL-4 par ces cellules chez les patients diabétiques par rapport aux sujets contrôles [23]. II faut toutefois noter que des résultats divergents ont été publiés en ce qui concerne la fréquence des sous-populations et la production de cytokines par les lymphocytes iNKT dans le sang des sujets ayant un diabète de type 1 . Ces différences pourraient en partie s'expliquer par la très grande hétérogénéité de la fréquence des lymphocytes iNKT chez l'homme et par les différentes techniques utilisées pour l'analyse de cette population [4]. Par conséquent, il serait intéressant de réitérer ces études avec un grand nombre de patients et une méthode standardisée.

Par ailleurs, du fait de l'impossibilité de pratiquer des biopsies de pancréas chez l'homme, la présence de lymphocytes iNKT dans ce tissu n'a pas été analysée. Cependant, la création du programme nPOD (network for pancreatic organ donors with diabetes) pourrait permettre l'étude des lymphocytes iNKT à partir d'une banque de pancréas de patients qui avaient un diabète de type 1 . 


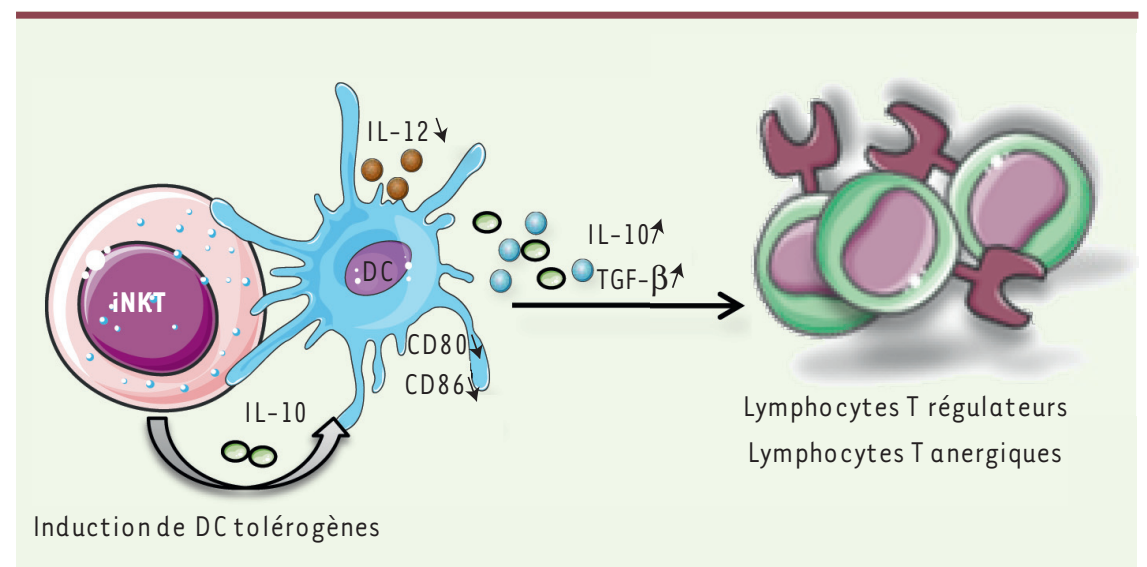

Figure 3. Régulation du diabète de type 1 par le biais de cellules dendritiques. Les lymphocytes iNKT peuvent induire des cellules dendritiques (DC) tolérogènes qui expriment faiblement CD80 et CD86 et secrètent de I'IL-10 et/ou du TGF $\beta$ (transforming growth factor $\beta$ ), favorisant la conversion des lymphocytes $T$ naifs en lymphocytes $T$ régulateurs ou anergiques. Cette induction est indépendante de la sécrétion d'IL-4 par les lymphocytes iNKT, mais dépendante des contacts cellulaires entre les lymphocytes iNKT et les DC et/ou de la production d'IL-10 par les lymphocytes iNKT.

\section{Rôle protecteur des lymphocytes iNKT dans le diabète lors d'une infection virale}

La dualité fonctionnelle des lymphocytes iNKT nous a incités à étudier leur rôle régulateur dans le diabète de type 1 au cours des infections virales. En effet, parallèlement aux études qui ont montré le rôle tolérogène des lymphocytes iNKT dans le diabète de type 1 et dans d'autres pathologies auto-immunes, plusieurs laboratoires ont observé un rôle activateur des lymphocytes iNKT au cours des infections [3]. Lors d'infections virales, bactériennes ou parasitaires, les lymphocytes iNKT sont activés très rapidement et stimulent à leur tour les réponses immunitaires antiinfectieuses. Par conséquent, une question fondamentale se pose: les lymphocytes iNKT peuvent-ils effectuer ces deux fonctions, maintien de la tolérance immunitaire et réponse anti-infectieuse efficace, en même temps et sans préjudice pour l'intégrité de l'organisme? Le modèle privilégié pour répondre à cette question a été le diabète induit par une infection par le virus de la chorioméningite lymphocytaire (LCMV). Dans ce contexte, les lymphocytes iNKT favorisent la réponse immunitaire antivirale, tout en protégeant les souris contre le développement du diabète $[24,25]$. Dans la rate, les cellules iNKT activent les cellules dendritiques conventionnelles qui produisent de l'IL-12 et induisent une forte réponse CD8 anti-LCMV, indispensable à l'élimination du virus par la lyse des cellules infectées. Dans le pancréas, les lymphocytes iNKT n'interagissent pas avec les cellules dendritiques conventionnelles, mais avec les cellules dendritiques plasmacytoïdes ( $p D C)$. Au cours de l'infection par le LCMV, les lymphocytes iNKT pancréatiques sont activés et ils induisent le recrutement des pDC dans ce tissu. De plus, les lymphocytes iNKT activent la production par les pDC de grandes quantités d'IFN $\alpha$, qui inhibe la réplication virale dans le pancréas et limite ainsi la destruction du tissu pancréatique [26]. Dans un second temps, les pDC migrent dans les ganglions pancréatiques où elles produisent du TGF $\beta$ et induisent la conversion des lymphocytes $T$ naïfs en cellules $T$ régulatrices (FoxP3 ${ }^{+}$) (Figure 3). Ensuite, ces cellules T régulatrices migrent dans le pancréas où elles secrètent du TGF $\beta$ qui inhibe les lymphocytes T anti-cellules $\beta$ pancréatiques. Ces deux mécanismes complémentaires mettent en jeu les lymphocytes iNKT et les pDC qui contrôlent la réplication virale, tout en prévenant l'apparition du diabète [24]. II est intéressant de noter que ces mécanismes régulateurs sont également impliqués dans l'inhi- bition du diabète spontané après infection par le LCMV. II était connu depuis de nombreuses années que certaines infections inhibaient le développement du diabète de type 1 dans les modèles animaux [25], et nos travaux ont révélé les mécanismes cellulaires et moléculaires impliqués dans la protection induite par le LCMV. II est donc très intéressant de constater que l'activation du système immunitaire peut être protectrice dans les maladies auto-immunes. Par ailleurs, nos résultats préliminaires sur le rôle des lymphocytes iNKT lors d'une infection par le virus Coxsackie B4 montrent également que les lymphocytes protègent contre le développement du diabète induit par cette infection virale. Toutefois, les mécanismes de protection sont différents de ceux mis en jeu lors de l'infection par le LCMV. L'ensemble de ces résultats est encourageant pour le développement de stratégies thérapeutiques contre le diabète de type 1, au moyen de l'activation des lymphocytes iNKT.

\section{Manipulation thérapeutique des lymphocytes iNKT dans le diabète}

\section{Activation des lymphocytes iNKT par des ligands spécifiques}

\section{Traitement par l' $\alpha$ GalCer}

Le glycolipide $\alpha$ GalCer, initialement purifié à partir de l'éponge marine Agelas mauritianus, stimule spécifiquement les lymphocytes iNKT chez l'homme et la souris [3]. La reconnaissance du complexe CDldaGalCer par le TCR des lymphocytes iNKT aboutit à la production rapide de cytokines. Des injections multiples d' $\alpha$ GalCer à des souris NOD prédiabétiques préviennent le développement du diabète [27]. Ce traitement induit l'accumulation des lymphocytes iNKT produisant de I'IL-4 dans les îlots pancréatiques ainsi que dans les ganglions pancréatiques. Cette cytokine pourrait être impliquée dans la protection contre le diabète. Cette 


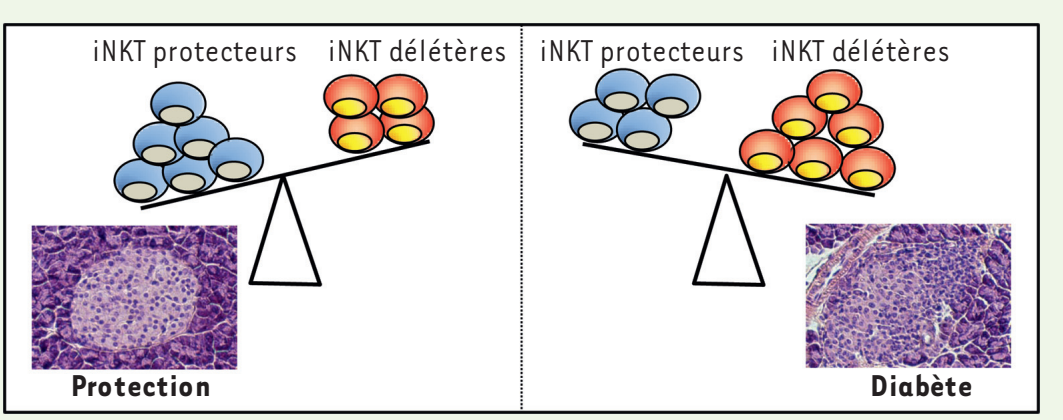

Figure 4. Rôle ambivalent des lymphocytes iNKT dans le diabète de type 1. Chez la souris NOD, une sous-population de lymphocytes iNKT (iNKT CD4 $4^{+}$) exerce un rôle protecteur, alors qu'une autre sous-population (CD4- iNKT17) stimule la maladie. Par conséquent, la protection (gauche) ou la stimulation (droite) de la réponse auto-immune anti-îlot(s) pourrait être due à un déséquilibre quantitatif entre ces différentes sous-populations. Photographies : coupe de pancréas en coloration H\&E (hématoxyline-éosine) montrant un ilôt pancréatique sain (gauche : protection) ou infiltré (droite : diabète). très faible production de cytokines, dont I'IL-4, par les lymphocytes iNKT. II est particulièrement intéressant d'observer que cette molécule $\mathrm{Cl6:0}$ prévient plus efficacement le diabète chez les souris NOD que l'aGalCer [29]. À nouveau, ces données illustrent le rôle régulateur des lymphocytes iNKT indépendamment de I'IL-4. Les mécanismes de prévention mis en jeu par un traitement avec du C16:0 sont en cours d'analyse dans notre laboratoire. Par ailleurs, il serait important de tester l'efficacité de cette molécule dans la prévention du diabète induit par des infections virales, le LCMV et également le virus Coxsackie B4 qui a été proposé comme agent étiologique dans le diabète de type 1 [30]. prévention n'est plus observée dans les souris NOD déficientes en IL-4, ce qui démontre le rôle régulateur de cette cytokine. Toutefois, comme décrit précédemment, la production d'IL-4 n'est probablement pas le seul paramètre impliqué dans la prévention du diabète par l'activation des lymphocytes iNKT. Plusieurs équipes ont observé qu'un traitement itératif par l' $\alpha$ GalCer induit une modification de la fonction des cellules présentatrices d'antigène. Ainsi, des cellules dendritiques tolérogènes, de phénotype $\operatorname{CD} 11 c^{+} / \operatorname{CD} 8 \alpha^{-}$, s'accumulent dans les ganglions pancréatiques. Ces cellules produisent des quantités réduites d'IL-12, une cytokine pro-Thl favorisant les réponses T diabétogènes. Par ailleurs, ces cellules dendritiques expriment aussi plus faiblement les récepteurs de costimulation CD80 et CD86 que les cellules de souris ayant reçu une seule injection d' $\alpha$ GalCer [28]. Toutes ces modifications des cellules dendritiques peuvent induire une absence de réponse immune ou des réponses régulatrices. De façon intéressante, l'effet suppresseur de ce type de traitement semble spécifique des lymphocytes T autoréactifs anti-cellules $\beta$ pancréatiques, car la stimulation des splénocytes par de la concanavaline $A$, un activateur polyclonal des lymphocytes T, induit leur production d'IL-4 et d'IFN $\gamma$. Malgré ses effets bénéfiques contre le développement du diabète, il a été montré que l' $\alpha$ GalCer pourrait exacerber d'autres pathologies autoimmunes, comme le lupus [4]. De plus, les traitements répétés par de l'aGalCer entraînent la sécrétion d'IL-4 par les lymphocytes iNKT, et cette cytokine pourrait favoriser certaines réactions allergiques (comme l'asthme) chez des sujets prédisposés. Ces données suggèrent qu'un traitement par l' $\alpha$ GalCer ne serait pas le plus approprié, et de nouveaux agonistes des lymphocytes iNKT sont actuellement produits et testés dans différents modèles animaux.

\section{Les analogues de l' $\alpha$ GalCer}

Des modifications structurales de l' $\alpha$ GalCer influencent le profil des cytokines produites par les lymphocytes iNKT. Un analogue de l' $\alpha$ GalCer, dénommé C16:0, possède la particularité de n'induire qu'une

\section{Augmentation du nombre de lymphocytes iNKT}

Chez la souris, nous avons démontré que l'augmentation du nombre de lymphocytes iNKT permet de réduire significativement la progression du diabète de type 1 [7]. Augmenter le nombre de lymphocytes iNKT semble donc une stratégie intéressante à transposer à l'homme.

\section{Expansion in vitro des lymphocytes iNKT}

Plusieurs laboratoires ont développé des méthodes in vitro permettant d'accroître le faible nombre de lymphocytes iNKT humains présents dans le sang périphérique. Un essai clinique de phase 1 a montré que l'injection de lymphocytes iNKT obtenus par expansion in vitro était bien tolérée et sans danger [31]. Cette stratégie présente l'avantage de pouvoir activer puis sélectionner spécifiquement certaines sous-populations de lymphocytes iNKT. Par analogie avec les données chez la souris, on pourrait proposer de sélectionner, grâce à leurs marqueurs de surface, les lymphocytes iNKT CD4 ${ }^{+}$au fort pouvoir régulateur, et d'exclure les lymphocytes iNKT17 qui ont un rôle délétère dans le diabète [4].

\section{Augmenter la présentation antigénique}

Dans le diabète de type 1 , le rôle des antigènes du soi (glycolipides) dans l'activation des lymphocytes iNKT reste encore inconnu. Récemment, il a été montré que certaines infections bactériennes induisent l'expression d'enzymes, comme la glucosylcéramide synthase, qui permettent la synthèse de glycolipides du soi. Ces glycolipides sont ensuite présentés aux lymphocytes iNKT et induisent leur prolifération [32]. II serait intéressant d'induire l'expression de ces enzymes chez les patients diabétiques afin d'augmenter le nombre de 


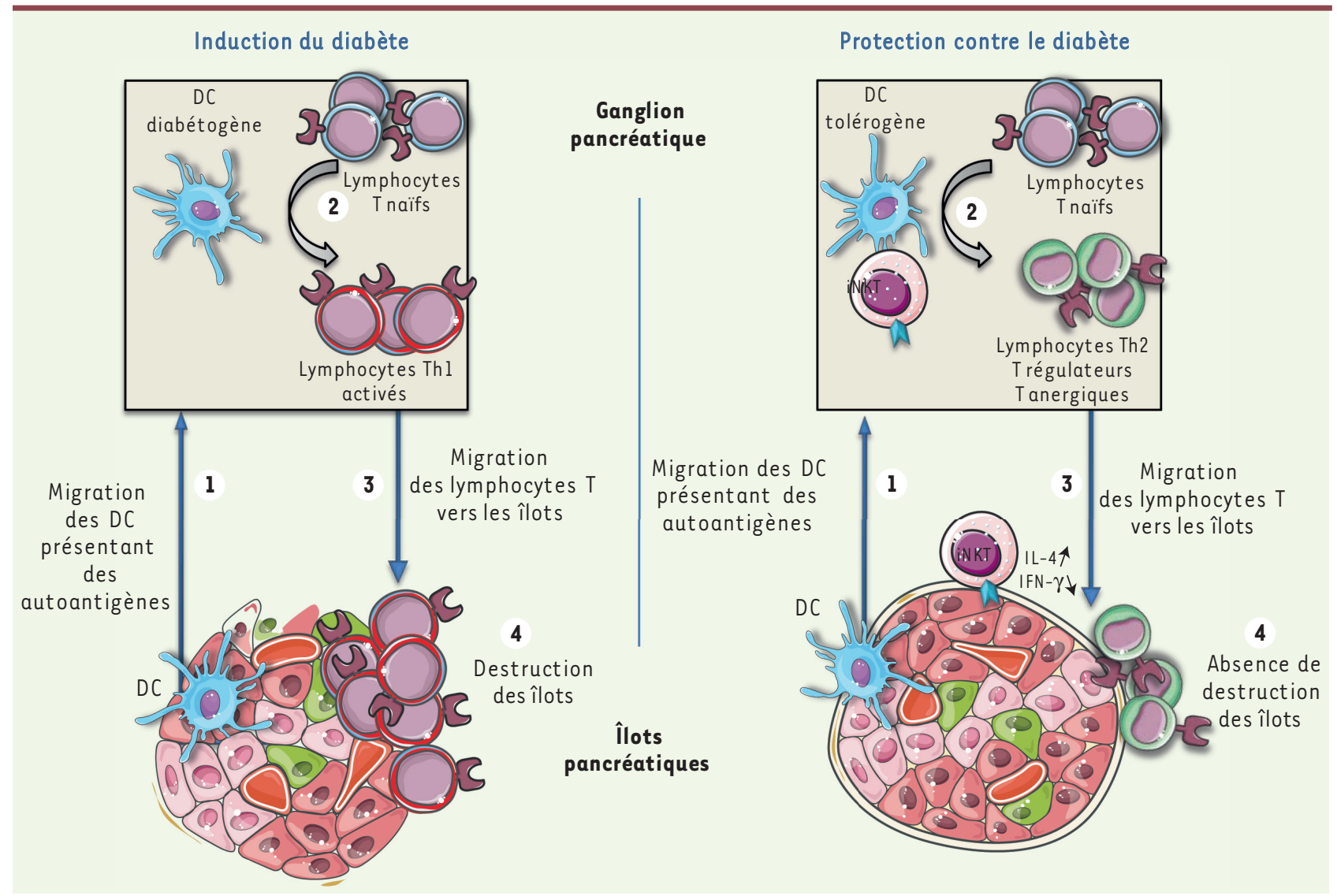

Figure 5. Mécanismes de régulation du diabète de type 1 par les lymphocytes iNKT. Au cours de l'induction du diabète, les cellules dendritiques (DC) diabétogènes migrent des îlots pancréatiques vers les ganglions drainant le pancréas (gauche). Dans les ganglions, les DC activent des lymphocytes T naïfs anti-îlots qui deviennent des lymphocytes Thl. Ces derniers migrent vers le pancréas où ils détruisent les cellules $\beta$, induisant ainsi le diabète. Toutefois, les lymphocytes iNKT peuvent induire des DC tolérogènes qui favorisent la différenciation des lymphocytes T naïfs en lymphocytes Th2, régulateurs ou anergiques (droite). Ces lymphocytes migrent vers le pancréas, mais ne détruisent pas les îlots.

lymphocytes iNKT. Cependant, il faudrait au préalable vérifier que cette technique augmente sélectivement les lymphocytes iNKT aux propriétés régulatrices et non pas ceux qui auraient un rôle délétère, comme les iNKT17.

\section{Conclusion}

Un ensemble de données démontrent donc le rôle régulateur des lymphocytes iNKT dans les modèles animaux du diabète de type 1 (Figure 5). Des études in vitro avec des lymphocytes iNKT humains confortent le rôle régulateur de cette population lymphocytaire chez I'homme [33]. De plus, les premiers essais cliniques basés sur la manipulation des lymphocytes iNKT ont démontré l'absence de toxicité de ces cellules. Il est raisonnable de penser que la production et la sélection de nouveaux agonistes des lymphocytes iNKT devraient permettre de proposer des composés susceptibles d'être testés dans des essais thérapeutiques de prévention du diabète de type 1 . Les recherches futures doivent avoir pour objectif une meilleure connaissance de cette population de cellules particulières, pour utiliser leur fonction régulatrice dans un but thérapeutique. $\diamond$

\section{SUMMARY}

Regulatory role of NKT cells in the prevention of type 1 diabetes

Type 1 diabetes is an autoimmune disease resulting from the destruction of pancreatic $\beta$ cells by the immune system. NKT cells are innate-like T cells that can exert potent immuno-regulatory functions. The regulatory role of NKT cells was initially proposed after the observed decreased frequency of this subset in mouse models of type 1 diabetes, as well as in patients developing various autoimmune pathologies. Increasing NKT cell frequency and function prevent the development of type 1 diabetes in mouse models. Several mechanisms including IL-4 and IL-10 production by NKT cells and the accumulation of tolerogenic dendritic cells are critical for the dampening of pathogenic anti-islet T cell responses by NKT cells. Importantly, these cells can at the same time prevent diabetes and promote efficient immune responses against infectious agents. These results strengthen 
the potential role of NKT cells as a key target for the development of therapeutic strategies against type 1 diabetes. $\diamond$

\section{REMERCIEMENTS}

Ce travail a été financé par l'ANR-09-GENO-023 et laboratoire d'excellence INFLAMEX, ainsi que le ministère de l'Éducation nationale et la région île-deFrance (CORDDIM), un contrat hospitalier de recherche translationnelle (AL). Illustrations réalisées grâce à Servier Medical Art.

\section{LIENS D'INTÉRÊT}

Les auteurs déclarent n'avoir aucun lien d'intérêt concernant les données publiées dans cet article.

\section{RÉFÉRENCES}

1. Lehuen A, Diana J, Zaccone P, Cooke A. Immune cell crosstalk in type I diabetes. Nat Rev Immunol $2010 ; 10: 501-13$.

2. Diana J, Simoni Y, Furio L, et al. Crosstalk between neutrophils, B-la cells and plasmacytoid dendritic cells initiates autoimmune diabetes. Nat Med $2013 ; 19: 65-73$.

3. Bendelac A, Savage PB, Teyton L. The biology of NKT cells. Annu Rev Immunol 2007 ; 25 : 297-336.

4. Simoni Y, Diana J, Ghazarian L, et al. Therapeutic manipulation of natural killer (NK) T cells in autoimmunity: are we close to reality? Clin Exp Immunol 2013 ; 171 : 8-19.

5. Gombert JM, Herbelin A, Tancrede-Bohin $\varepsilon$, et al. Early quantitative and functional deficiency of $\mathrm{NKI}^{+}$-like thymocytes in the NOD mouse. Eur J Immunol $1996 ; 26: 2989-98$.

6. Hammond KJ, Pellicci DG, Poulton LD, et al. CDld-restricted NKT cells: an interstrain comparison. JImmunol $2001 ; 167: 1164-73$.

7. Lehuen A, Lantz 0 , Beaudoin L, et al. Overexpression of natural killer T cells protects Valphal4 Jalpha281 transgenic nonobese diabetic mice against diabetes. J Exp Med 1998 ; 188 : 1831-9.

8. Falcone M, Facciotti F, Ghidoli N, et al. Up-regulation of CDld expression restores the immunoregulatory function of NKT cells and prevents autoimmune diabetes in nonobese diabetic mice. J Immunol $2004 ; 172$ : 5908-16.

9. Wang B, Geng YB, Wang CR. CDI-restricted NK T cells protect nonobese diabetic mice from developing diabetes. J Exp Med $2001 ; 194$ : 313-20.

10. Shi FD, Flodstrom M, Balasa B, et al. Germ line deletion of the CDI locus exacerbates diabetes in the NOD mouse. Proc Natl Acad Sci USA $2001 ; 98: 6777-82$

11. Rapoport MJ, Jaramillo A, Zipris D, et al. Interleukin 4 reverses $T$ cell proliferative unresponsiveness and prevents the onset of diabetes in nonobese diabetic mice. J Exp Med 1993 $178: 87-99$.

12. Laloux V, Beaudoin L, Jeske $D$, et al. NK T cell-induced protection against diabetes in V alpha 14-J alpha 281 transgenic nonobese diabetic mice is associated with a Th2 shift circumscribed regionally to the islets and functionally to islet autoantigen. J Immunol $2001 ; 166: 3749-56$.

13. Hammond KJ, Poulton LD, Palmisano LJ, et al. alpha/beta-T cell receptor (TCR)+CD4-CD8- (NKT) thymocytes prevent insulin-dependent diabetes mellitus in nonobese diabetic (NOD)/Lt mice by the influence of interleukin (IL)-4 and/or IL-10. J Exp Med 1998 ; 187 : 1047-56.

14. Chen YG, Choisy-Rossi CM, Holl TM, et al. Activated NKT cells inhibit autoimmune diabetes through tolerogenic recruitment of dendritic cells to pancreatic lymph nodes. J Immunol 2005; 174 1196-204.

15. Beaudoin L, Laloux V, Novak J, et al. NKT cells inhibit the onset of diabetes by impairing the development of pathogenic T cells specific for pancreatic beta cells. Immunity $2002 ; 17: 725-36$.

16. Naumov YN, Bahjat KS, Gausling R, et al. Activation of CDld-restricted T cells protects NOD mice from developing diabetes by regulating dendritic cell subsets. Proc Natl Acad Sci USA 2001 ; 98 : 13838-43.
17. Novak J, Beaudoin L, Park S. Prevention of type 1 diabetes by invariant NKT cells is independent of peripheral CDId expression. J Immunol 2007 . 178:1332-40.

18. Novak J, Beaudoin L, Griseri T, Lehuen A. Inhibition of T cell differentiation into effectors by NKT cells requires cell contacts. J Immunol 2005 ; 174 : 1954-61.

19. Michel ML, Keller AC, Paget C, et al. Identification of an IL-17-producing NK1.1(neg) iNKT cell population involved in airway neutrophilia. J Exp Med $2007 ; 204$ : 995-1001.

20. Simoni $Y$, Gautron AS, Beaudoin L, et al. NOD mice contain an elevated frequency of iNKT17 cells that exacerbate diabetes. Eur J Immunol 2011 ; $41: 3574-85$

21. Wilson SB, Kent SC, Patton KT, et al. Extreme Thl bias of invariant Valpha24JalphaQ T cells in type 1 diabetes. Nature $1998 ; 391: 177-81$.

22. Kis J, Engelmann P, Farkas $K$, et al. Reduced $C^{2} 4^{+}$subset and Thl bias of the human iNKT cells in Type 1 diabetes mellitus. J Leukoc Biol 2007 ; 81 : 654-62.

23. Kent SC, Chen Y, Clemmings SM, et al. Loss of IL-4 secretion from human type la diabetic pancreatic draining lymph node NKT cells. J Immunol 2005 ; $175:$ 4458-64.

24. Diana J, Brezar V, Beaudoin L, et al. Viral infection prevents diabetes by inducing regulatory T cells through NKT cell-plasmacytoid dendritic cell interplay.J Exp Med 2011 ; 208 : 729-45.

25. Oldstone MB. Prevention of type I diabetes in nonobese diabetic mice by virus infection. Science $1988 ; 239: 500-2$

26. Diana J, Griseri T, Lagaye $S$, et al. NKT cell-plasmacytoid dendritic cell cooperation via $0 \times 40$ controls viral infection in a tissue-specific manner. Immunity 2009; $30: 289-99$.

27. Sharif S, Arreaza GA, Zucker P, et al. Activation of natural killer T cells by alpha-galactosylceramide treatment prevents the onset and recurrence of autoimmune Type 1 diabetes. Nat Med 2001 ; 7 : 1057-62.

28. Wang J, Cho S, Ueno A, et al. Ligand-dependent induction of noninflammatory dendritic cells by anergic invariant NKT cells minimizes autoimmune inflammation. J Immunol 2008 ; 181 : 2438-45.

29. Blumenfeld HJ, Tohn R, Haeryfar SM, et al. Structure-guided design of an invariant natural killer T cell agonist for optimum protection from type 1 diabetes in non-obese diabetic mice. Clin Exp Immunol 2011 ; 166 : 121-33.

30. Ghazarian L, Diana J, Simoni Y, et al. Prevention or acceleration of type 1 diabetes by viruses. Cell Mol Life Sci $2013 ; 70: 239-55$.

31. Motohashi S, Ishikawa A, Ishikawa $\varepsilon$, et al. A phase I study of in vitro expanded natural killer T cells in patients with advanced and recurrent nonsmall cell lung cancer. Clin Cancer Res $2006 ; 12: 6079-86$.

32. Brennan PJ, Tatituri RV, Brigl M, et al. Invariant natural killer T cells recognize lipid self antigen induced by microbial danger signals. Nat Immunol 2011 ; $12: 1202-11$

33. Hegde S, Lockridge JL, Becker YA, et al. Human NKT cells direct the differentiation of myeloid APCs that regulate T cell responses via expression of programmed cell death ligands. J Autoimmun $2011 ; 37$ : 28-8.

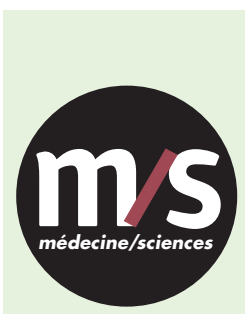

Grâce à $\mathrm{m} / \mathrm{s}$, vivez en direct les progrès

Tarifs d'abonnement $\mathrm{m} / \mathrm{s}-2013$ des sciences biologiques et médicales

\section{Abonnez-vous}

à médecine/sciences

Bulletin d'abonnement page 702 dans ce numéro de $\mathrm{m} / \mathrm{s}$
TIRÉS À PART

A. Lehuen

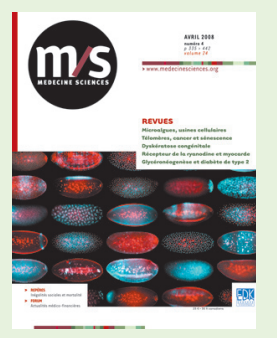

